\title{
RELIABILITY OF PRESSURE SENSORS TO MEASURE WAVE HEIGHT IN THE SHOALING REGION
}

\author{
Massimiliano Marino ${ }^{1}$, Iván Cáceres Rabionet ${ }^{2}$, Rosaria Ester Musumeci ${ }^{1}$, Enrico Foti $^{1}$
}

\begin{abstract}
A comparison between a range of transfer functions to recover wave height from pressure sensors data is presented. The analysis is carried out by means of a large-scale wave flume experimental dataset, in which resistive, acoustic and pressure gauges recovered wave height are compared as the waves travel from intermediate waters, to the shoaling region and finally into the surf zone. All the considered transfer functions result adequate in recovering wave height in intermediate waters, becoming gradually less accurate as the steepness of the wave increases in the shoaling region and in the surf zone. The accuracy of the compared transfer functions is assessed by means of an ensemble wave height based deviation.
\end{abstract}

Keywords: wave measurements; linear wave theory; surf zone

\section{INTRODUCTION}

Pressure sensors are widely used instruments to measure wave height both in laboratory and in field contexts. A commonly used relation to obtain wave height from pressure records is the one provided by linear wave theory, whose accuracy in recovering water elevation has been investigated by many. Hom-ma et al. (1967), Cavaleri et al. (1978) and Biesel (1982) found a significant difference between linear wave theory predictions and experimental observations; in order to account for that, a correction factor must be applied to the measured wave height. Bishop and Donelan (1987) by means of a large-scale flume laboratory campaign dataset and a review of the at-the-time existing methods, affirmed that linear wave theory returns reliable estimates of wave height within an error of 5\% in deep and intermediate waters. Nevertheless, they concluded that pressure response in shallow waters needs to be corrected due to the presence of wave nonlinearities. Indeed, the occurrence of shoaling waves in shallow waters, whose nonlinear components become way more significant as the wave travels towards the coast, determines wave characteristics to largely differ from the one predicted by linear wave theory.

Nonlinear effects are usually considered in wave theory by using Stokes expansion to obtain higher order correction terms (Kuo and Chiu, 1994; Chen, 2000). Tsai et al. (2005) assessed some of these nonlinear methods, evaluating their accuracy in recovering shoaling wave height and shape, showing that in certain conditions they may be even less accurate then linear wave theory. Nevertheless, they conclude, a nonlinear correction in shallow waters and surf zone is crucial.

Despite the acknowledged importance of using nonlinear methods, recent field and laboratory investigations still use linear wave theory derived formulas to obtain wave height from pressure records in shallow waters (Van Rijn et al., 2000; Inch, 2014).

In the light of the above, the present work aims to assess the performance of a range of commonly used transfer functions, in order to evaluate their accuracy in recovering wave height from pressure records as the wave travels towards the coast, inside and outside the shoaling region. A comparison between resistive, acoustic and pressure sensor data obtained from laboratory experiments is carried out in a large-scale flume. This allows to reduce the influence of scale effects, particularly at breaking point and further onshore. Data are subsequently analyzed in order to understand in what conditions the considered transfer functions become inaccurate.

The paper is organized as follows: in the first section the experimental setup used during the investigation and the experimental procedure are described in detail; in the second section different transfer functions are briefly introduced; in the third section results in terms of ensemble wave height and steepness are shown, in order to compare the accuracy of the considered transfer functions as steepness increases. A concluding section closes the work.

\footnotetext{
${ }^{1}$ Department of Civil Engineering and Architecture, University of Catania, Catania, Italy

${ }^{2}$ Laboratori d'Enginyeria Maritima, Universitat Politécnica de Catalunya, Barcelona, Spain
} 


\section{EXPERIMENTAL SETUP}

A laboratory test campaign is carried out at the Canal d'Investigació i Experimentació Marítima (CIEM) of Universitat Politécnica de Catalunya (UPC) in Barcelona (Spain). The cIEM is a large-scale wave flume of $100 \mathrm{~m}$ length, $3 \mathrm{~m}$ width and $4.5 \mathrm{~m}$ depth. Its dimensions allow to work close to natural conditions, hence scale effects are considerably reduced.

The flume is provided at one side with a wedge-type wave generator which can generate both regular and irregular waves up to $0.9 \mathrm{~m}$ in height. The flume bottom is fixed and follows a sloping beach profile, in order to induce shoaling as the waves travel towards the beach. At the top of the slope, a beach bar is intended to induce breaking.

Three regions in the flume are defined: intermediate waters, shoaling region and surf zone. Shoaling region starts from shallow waters (depth equals to $L / 20$ for the longest considered wave) and ends at the highest point of the breaking bar.

A set of wave probes is positioned in the flume in order to measure waves, the entire system includes: 13 resistive gauges (WG), 11 acoustic gauges (AWG) and 11 pressure sensors (PPT). Flume profile and gauges deployment are shown in Figure 1.

In order to carry out the comparison of the measured wave height, gauges are arranged adjacent to one another and joined together in groups; every group includes one resistive wave gauge, one pressure sensor and one acoustic wave gauge. Six groups of adjacent gauges are arranged, two for every region. Position of the wave sensors in each group is shown in Table 1.

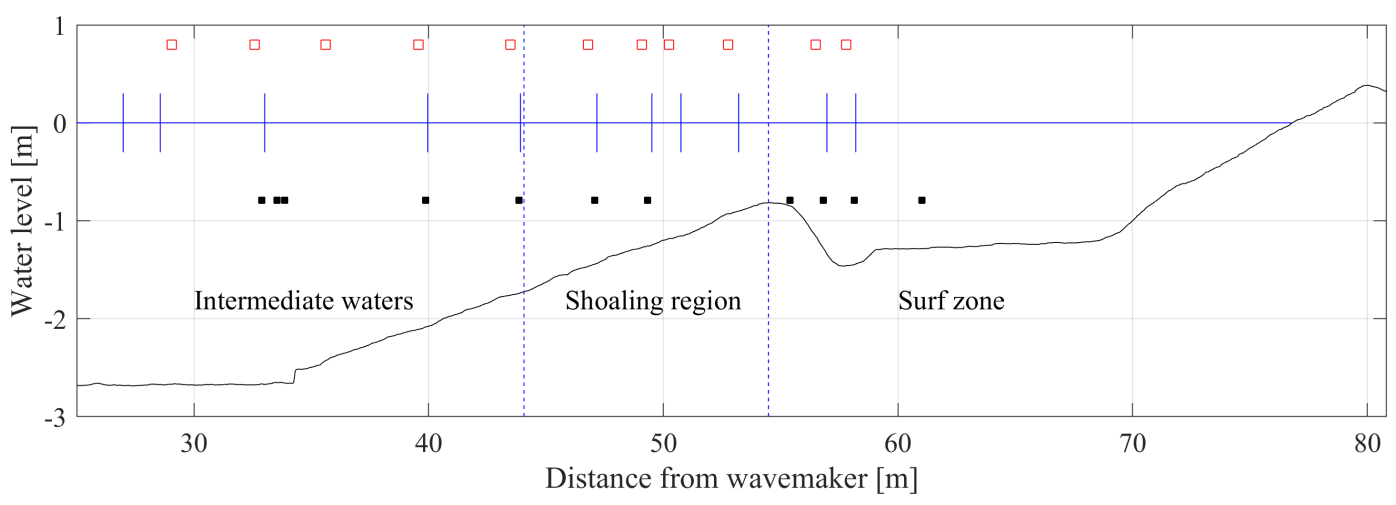

Figure 1: Wave gauges positioning along the flume. Continuous lines are wave gauges, red empty squares are the acoustic wave gauges and black full squares are pressure sensors.

Table 1: Gauges distance from the wavemaker.

\begin{tabular}{cccc}
\hline Group & WG position $[\mathrm{m}]$ & AWG position $[\mathrm{m}]$ & PPT position $[\mathrm{m}]$ \\
\hline 1 & 33.01 & 32.59 & 32.88 \\
2 & 39.96 & 39.87 & 39.87 \\
3 & 43.91 & 43.48 & 43.85 \\
4 & 50.75 & 56.81 & 49.32 \\
5 & 56.97 & 56.49 & 56.81 \\
6 & 58.20 & 57.79 & 58.13 \\
\hline
\end{tabular}

A total of 15 tests with different wave conditions, in terms of wave height $H$ and wave period $T$, have been conducted (Table 2). Still water level is the same for all tests and is equal to $2.65 \mathrm{~m}$ at the deepest point in the flume. All the generated waves are regular waves. 
Table 2: Wave height and period for all the experiments.

\begin{tabular}{ccccccccccccccccc}
\hline Test & & 1 & 2 & 3 & 4 & 5 & 6 & 7 & 8 & 9 & 10 & 11 & 12 & 13 & 14 & 15 \\
\hline$H$ & {$[m]$} & 0.4 & 0.4 & 0.4 & 0.4 & 0.5 & 0.5 & 0.5 & 0.5 & 0.6 & 0.6 & 0.6 & 0.7 & 0.3 & 0.2 & 0.2 \\
$T$ & {$[s]$} & 4.0 & 5.0 & 6.0 & 7.0 & 3.0 & 4.0 & 5.0 & 6.0 & 3.0 & 4.0 & 5.0 & 3.0 & 3.0 & 4.0 & 3.0 \\
\hline
\end{tabular}

\section{TRANSFER FUNCTIONS USED TO CORRECT THE PRESSURE SENSOR MEASUREMENTS}

The methods examined in the present work include the following transfer functions: (i) Kuo and Chiu (1994) empirical transfer function, (ii) Neumeier (2006) linear wave theory pressure correction, (iii) Oliveras et al. (2012) heuristic model, (iv) Inch (2014) linear wave theory cut-off frequency.

In particular, Kuo and Chiu (1994) propose a simple fully empirical transfer function obtained by laboratory experimental data, which is of the type:

$$
T F=\frac{p}{\rho g \eta}
$$

where $p$ is the measured pressure, $z$ is the pressure sensor depth of submergence, $\rho$ is water density, $g$ is gravitational acceleration, $\eta$ is the wave elevation and

$$
T F=\exp \left(-0.905 \frac{\omega^{2}|z|}{g}-0.027\right)
$$

where $\omega$ is the angular wave frequency. Neumeier and Amos (2006) within an experimental campaign apply a pressure correction to the linear wave theory based on Tucker and Pitt (2001). Linear wave theory formula for total pressure is:

$$
p(z)=\rho g z+\rho g K_{p} \eta
$$

where $K_{p}$ is the pressure response factor. Although the above relation is still largely used to obtain water surface elevation from pressure records many studies reveal that it shows inaccuracies in the presence of high frequency waves, due to the amplification operated by the pressure response factor $K_{p}$ (Bishop and Donelan, 1987); in that condition, the function returns inaccurate wave elevations. A filter is then necessary in order to apply pressure correction factor only within a certain frequency range, which is set by Neumeier (2006) as $0.05-0.33 \mathrm{~Hz}$.

Oliveras et al. (2012) propose a nonlinear nonlocal implicit relationship obtained from Euler's equation. Within the same investigation, they propose some lower order approximations. A good compromise between computational cost and accuracy is achieved by the following heuristic approximation:

$$
\eta=\frac{\mathcal{F}^{-1} \cosh (k h) \mathcal{F}\left\{\frac{p}{\rho g}\right\}}{1-\mathcal{F}^{-1}\left\{k \sinh (k h) \mathcal{F}\left\{\frac{p}{\rho g}\right\}\right.}
$$

where $k$ is the wave number, $h$ is the local water depth and $\mathcal{F}$ indicates Fast Fourier Transform.

Within an investigation on surf zone hydrodynamics, Inch (2014) use the following angular frequency cut-off formula by Aagaard et al. (2002):

$$
\omega_{c}=0.564 \pi \sqrt{\frac{g}{z}}
$$




\section{DATA ANALYSIS}

Wave elevation obtained from all the deployed instruments is examined considering the average wave measurements. Subsequently, an ensemble wave height based deviation to assess transfer function performance in recovering wave height is computed:

$$
D_{e, t f}=\left|1-\frac{H_{e, t f}}{H_{e, w g}}\right|
$$

in which $H_{e, t f}$ is the ensemble wave height obtained from the considered pressure transfer function and $H_{e, w g}$ is the ensemble wave height measured by resistive wave gauges. It must be stressed that this kind of evaluation assesses the ability of the transfer function to recover wave height, thus the performance of the considered transfer function in reconstructing wave shape, although qualitatively discussed here, is not considered in the final assessment.

Ensemble average wave recovered by resistive, acoustic and pressure gauges data are compared as the waves travel from intermediate waters to the shoaling region, and finally into the surf zone. Wave height measurements obtained from acoustic and resistive gauges are used as benchmark for the assessment of the pressure transfer functions accuracy. Figure 2 shows the ensemble average waves for Test 1 , group $1(H=$ $0.4 \mathrm{~m}, T=4.0 \mathrm{~s}, h=2.65 \mathrm{~m})$, thus in intermediate waters.
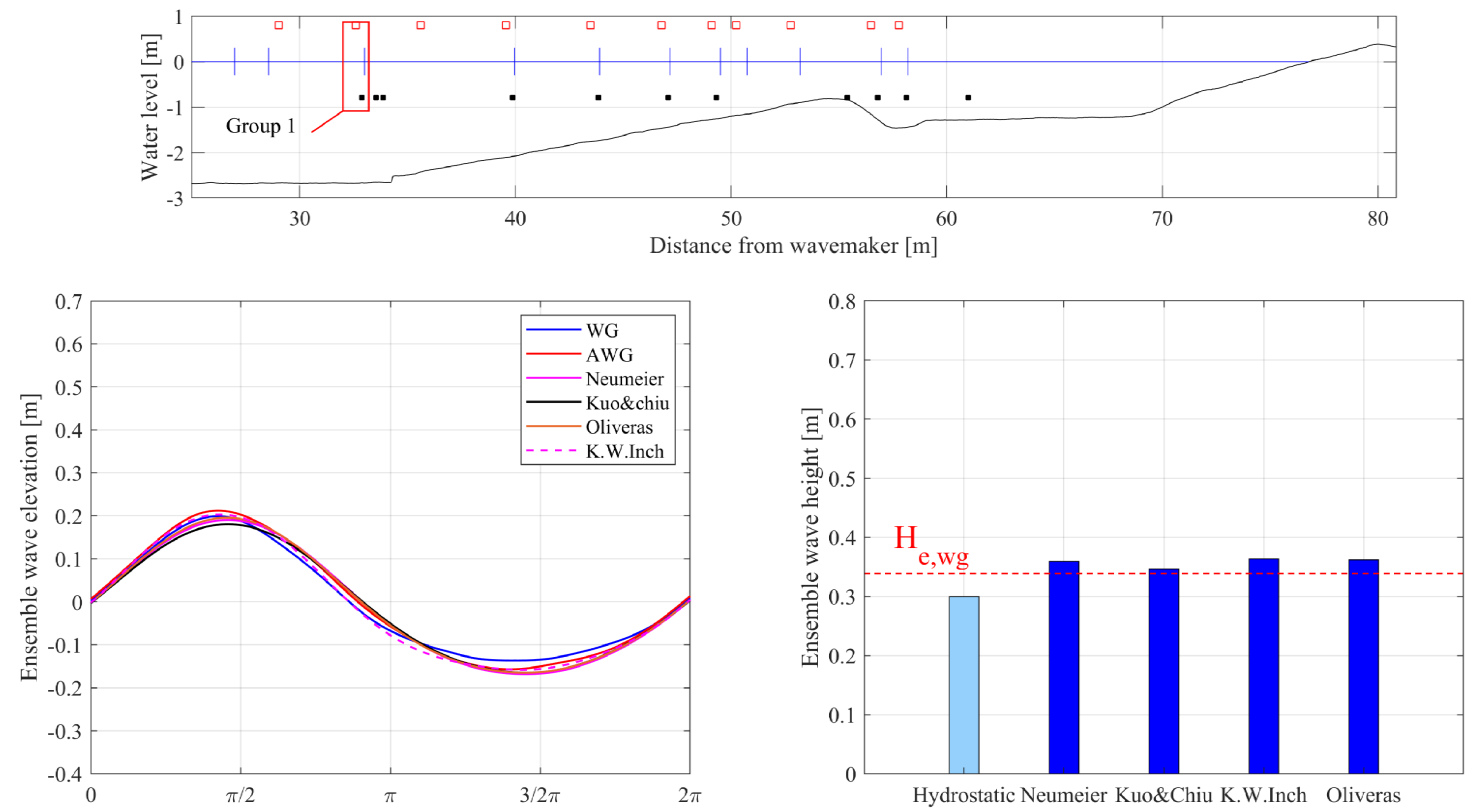

Figure 2: Ensemble wave height transfer function comparison in intermediate waters: (a) position of the gauge group in the flume, (b) ensemble wave comparison, (c) ensemble wave height comparison for Test 1 , group $1(\mathrm{H}=0.4 \mathrm{~m}, \mathrm{~T}=4.0 \mathrm{~s}, \mathrm{~h}=\mathbf{2 . 6 5} \mathrm{m})$.

Figure $2 \mathrm{a}$ shows the position of the group in the flume, Figure $2 \mathrm{~b}$ shows the ensemble average wave elevation obtained by resistive and acoustic sensors and the results of the four considered transfer functions, whereas Figure $2 \mathrm{c}$ shows the comparison between the ensemble average wave height obtained from all the transfer functions (blue bars) and the ensemble average wave height recovered by the resistive gauges (dashed line). The non-corrected hydrostatic pressure ensemble wave height is also plotted (light blue bar).

The wave ensemble comparison (Figure $2 \mathrm{a}$ and $\mathrm{b}$ ) shows that, in intermediate waters, all the considered transfer functions are able to accurately recover the wave height measured by resistive and acoustic gauges. Ensemble wave height deviation $D_{e, t f}$ for all the transfer functions is less than $5-10 \%$ in most of the cases, 
with the larger deviation observed for steeper waves. The non-corrected pressure sensor measurements significantly underestimate wave height already in intermediate waters.

Figure 3 shows the wave ensemble comparison for Test 12, group $4(H=0.7 \mathrm{~m}, T=3.0 \mathrm{~s}, h=1.74$ $\mathrm{m})$, thus into the shoaling region.
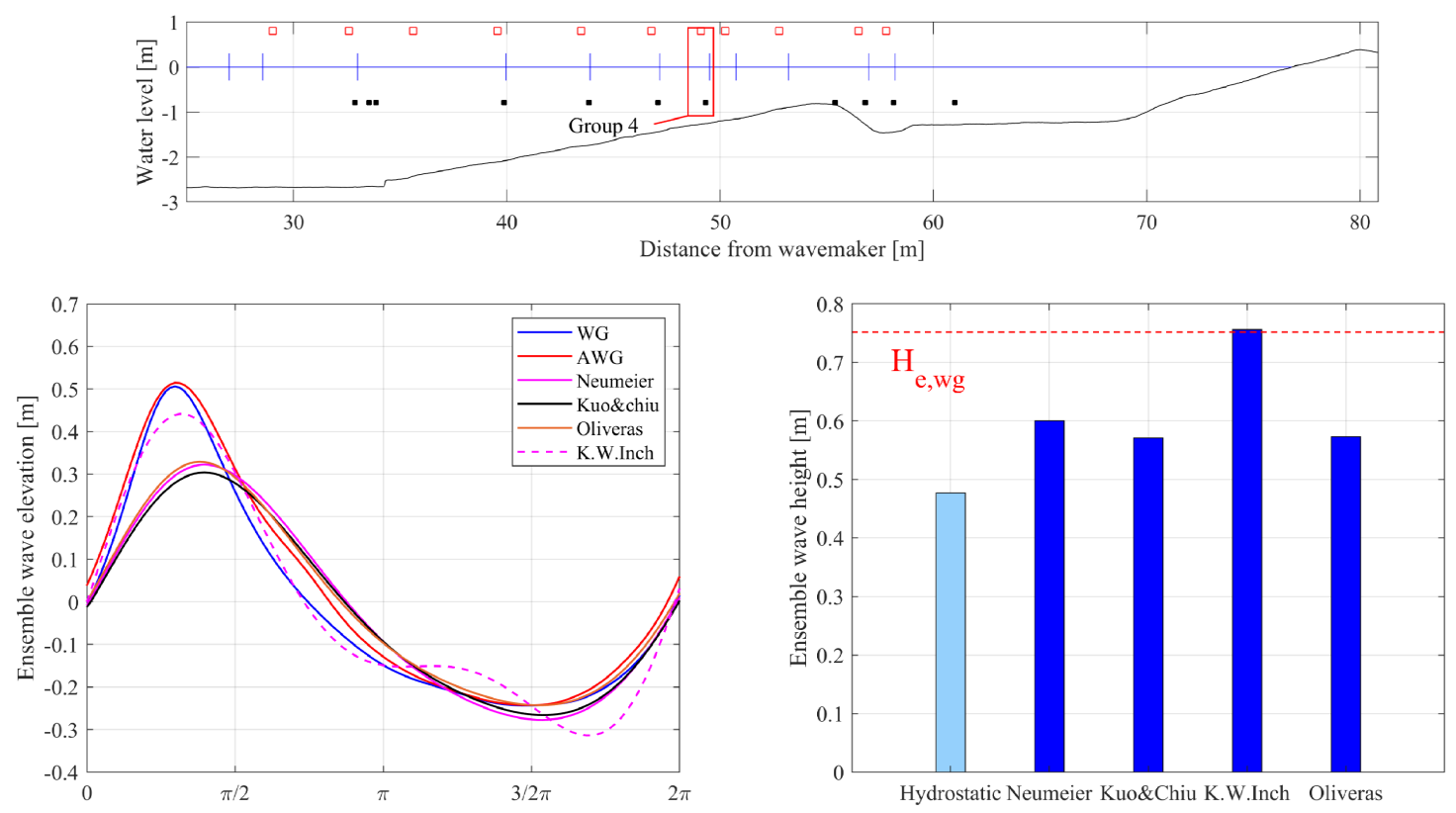

Figure 3: Ensemble wave height transfer function comparison in the shoaling region: (a) position of the gauge group in the flume, (b) ensemble wave comparison, (c) ensemble wave height comparison for test 12, group $4(\mathrm{H}=\mathbf{0 . 7} \mathrm{m}, \mathrm{T}=3.0 \mathrm{~s}, \mathrm{~h}=1.74 \mathrm{~m})$.

As the wave travels into shallow waters, wave-bottom interaction determines shoaling effects to become stronger, inducing the wave crest to become steeper as the wave trough widens. Figure 3 shows that three out of four investigated transfer functions, namely Neumeier, Kuo \& Chiu and Oliveras, behaves in a similar way. Indeed, none of them is able to return an accurate reconstruction of the wave crest height or shape, while reconstructing properly the wave trough. Ensemble wave height deviation $D_{e, t f}$ for these transfer function reaches values in the range of $20-25 \%$.

The K.W. Inch transfer function recovers significantly better both wave crest height and shape, with a deviation under $10 \%$ for most of the tests. Indeed, the higher K.W. Inch cut-off frequency, lets higher frequency components to be compensated by the pressure response factor, leading to a better reconstruction of the steeper crest. On the other hand, it induces an over amplification of nonlinear higher order harmonics that may appear in the trough. Thus, K.W. Inch transfer function recovers correctly wave height, however it leads to a not proper reconstruction of the wave shape in the trough.

Figure 4 shows wave ensemble comparison in the surf zone for Test 6 , group $6(H=0.5 \mathrm{~m}, T=4.0$ $\mathrm{s}, h=1.45 \mathrm{~m}$ ). Figure $4 \mathrm{~b}$ shows that, acoustic and resistive wave gauges no longer return comparable values of the ensemble wave elevation. Indeed, the splashes determined by breaking which occurs near the measurement area interfere with the signal sent by the acoustic wave transducer, resulting in the acoustic receiver to acquire the acoustic signal reflected back by wave splashes, rather than water surface. Acoustic wave gauges significantly overestimates wave height in the surf zone for all tests, thus the transfer function assessment is carried out by comparison with only the ensemble wave recovered by resistive gauges.

It must be stressed that in the surf zone, depending on the initial wave condition, breaking may occur up or downstream the measuring gauges, which instead are deployed at fixed locations along the flume for all tests, determining the presence of breaking and non-breaking wave tests. In the following examination 

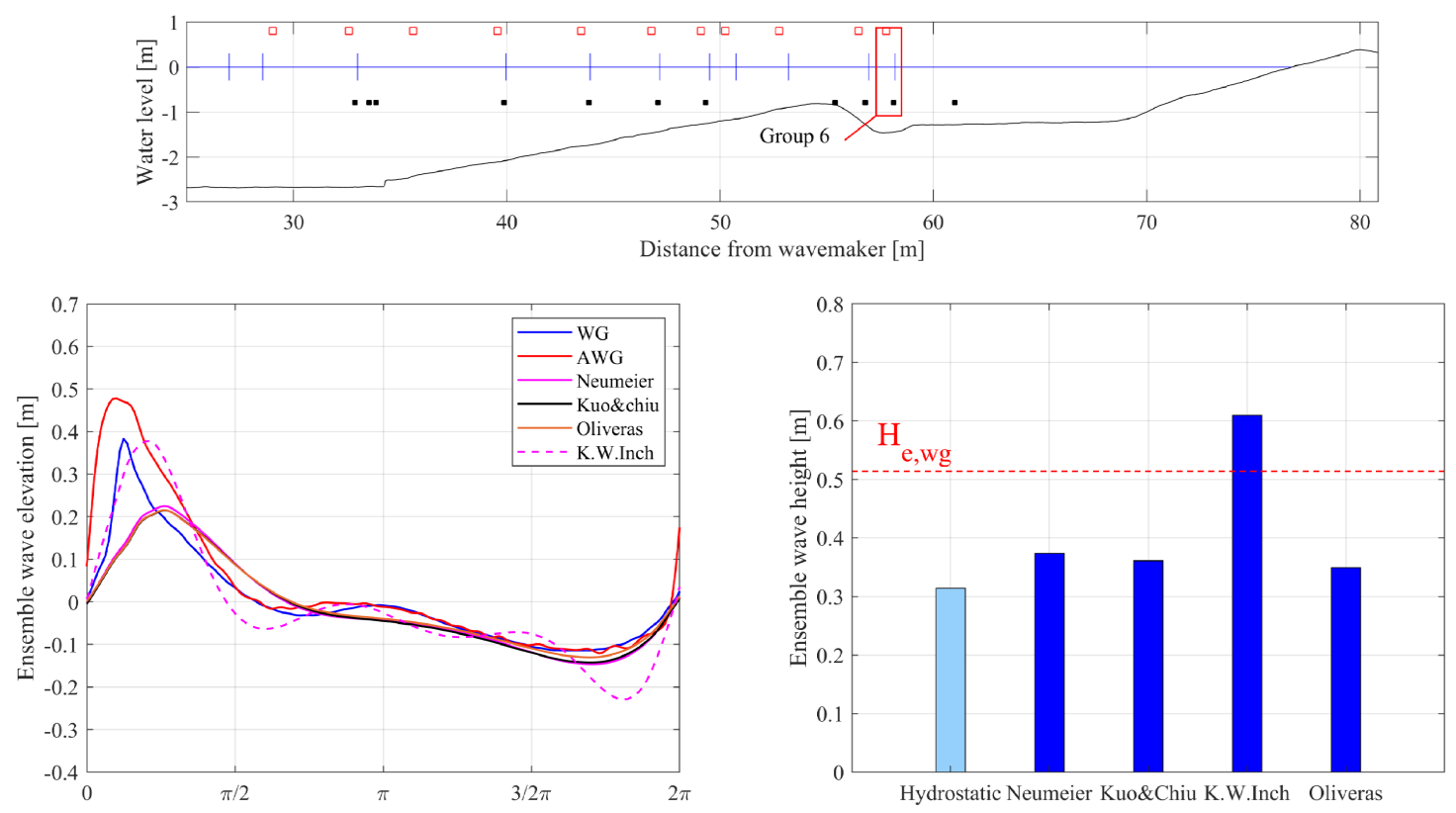

Figure 4: Ensemble wave height transfer function comparison in the surf zone: (a) position of the gauge group in the flume, (b) ensemble wave comparison, (c) ensemble wave height comparison for test 6 , group $6(\mathrm{H}=0.5 \mathrm{~m}, \mathrm{~T}=4.0 \mathrm{~s}, \mathrm{~h}=1.45 \mathrm{~m})$.

both conditions are considered.

Within the surf zone, Neumeier, Kuo \& Chiu and Oliveras show a slightly larger wave height underestimation; a deviation $D_{e, t f}$ in the range of $20-25 \%$ is observed. K.W. Inch transfer function higher frequencies amplification in the trough significantly increases in the surf zone, resulting in a significant overestimation of the wave height. $D_{e, t f}$ reaches values up to $40-45 \%$.

In the following we discuss how ensemble wave height recovered by pressure transfer functions deviates from the one recovered by resistive gauges as the wave steepness increase. Figure 5 shows how ensemble wave height recovered by Neumeier transfer function deviates from resistive gauges as the wave steepness increases for all tests. Results indicate that in intermediate waters (circles) the ratio between Neumeier ensemble wave height and resistive gauges wave height is near to unity, while in the shoaling region (triangles) Neumeier method deviates gradually as the wave steepness increases, deviating even more in the surf zone (squares).

Figure 6 shows that, in comparison with the Neumeier approach, K.W. Inch method provides better estimates of wave height in both intermediate waters and shoaling region. Nevertheless, even the latter method, leads to a significant wave height overestimation in the surf zone.

\section{CONCLUSIONS}

In the present work an ensemble wave height analysis from pressure sensor data is carried out, in order to evaluate the accuracy in recovering wave height of a range of pressure - surface elevation correction formulas. A laboratory experimental campaign in a large-scale flume is carried out, in which wave measurements are performed by means of resistive, acoustic and pressure sensors. The flume bottom profile allows the wave to travel in intermediate waters, then over a sloping bottom intended to induce shoaling, and finally to a beach bar. Data are furtherly analyzed by means of a wave ensemble wave height comparison between resistive, acoustic and pressure sensors recovered data, in which transfer function performance is assessed by means of an ensemble wave height deviation based coefficient. Four transfer functions in the assessment are considered, namely (i) Kuo and Chiu (1994) empirical transfer function, (ii) Neumeier 


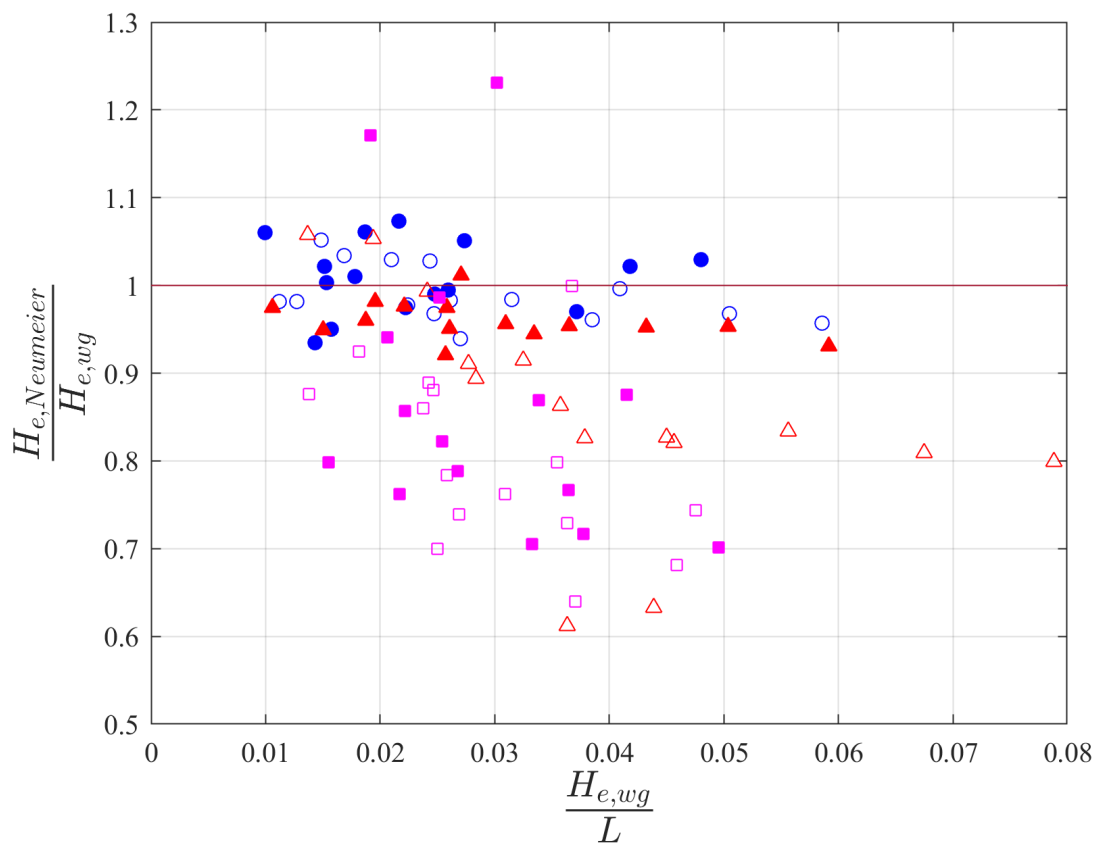

Figure 5: Ratio between Neumeier ensemble wave height and the resistive gauges ensemble wave height as the wave steepness increases in intermediate waters (circles), in the shoaling region (triangles) and in the surf zone (squares). Unfilled markers indicates groups 1, 3 and 5, filled markers indicates groups 2, 4 and 6.

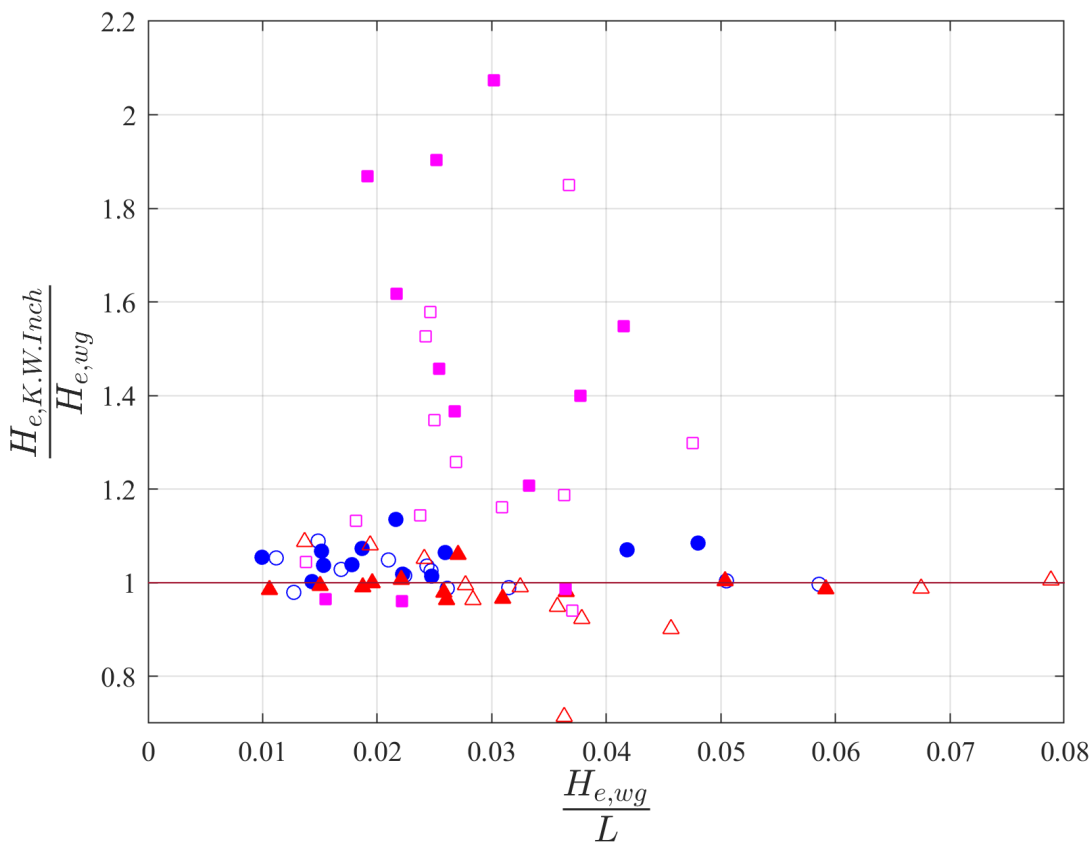

Figure 6: Ratio between K.W. Inch ensemble wave height and the resistive gauges ensemble wave height as the wave steepness increases in intermediate waters (circles), in the shoaling region (triangles) and in the surf zone (squares). Unfilled markers indicates groups 1, 3 and 5, filled markers indicates groups 2, 4 and 6. 
(2006) linear wave theory pressure correction, (iii) Oliveras et al. (2012) heuristic model, (iv) Inch (2014) linear wave theory cut-off frequency.

Results show that in intermediate waters all the considered transfer functions return reliable estimates of wave height, comparable to the ones recovered by resistive and acoustic wave gauges, although a slight underestimation for steeper waves is observed. In the shoaling region, three out of four transfer functions, namely Neumeier, Kuo \& Chiu, and Oliveras underestimate wave height whereas K.W. Inch method performs well as in intermediate waters, however returning a not correct shape reconstruction of the trough. In the surf zone, K. W. Inch method tends to significantly overestimate wave height due to higher order harmonics amplifications in the trough, whereas the other methods keeps underestimating ensemble wave height. Deviation $D_{e, t f}$ in the three regions averaged for all the tests is shown in Table 3.

Table 3: Transfer functions deviation averaged for all tests within the three regions

\begin{tabular}{lcccccc}
\hline & \multicolumn{2}{c}{ Intermediate waters } & \multicolumn{2}{c}{ Shoaling region } & \multicolumn{2}{c}{ Surf zone } \\
\hline Transfer function & Group 1 & Group 2 & Group 3 & Group 4 & Group 5 & Group 6 \\
\hline Neumeier & 0.04 & 0.04 & 0.04 & 0.16 & 0.20 & 0.19 \\
Kuo \& Chiu & 0.04 & 0.07 & 0.06 & 0.17 & 0.22 & 0.22 \\
Oliveras & 0.06 & 0.04 & 0.07 & 0.20 & 0.25 & 0.24 \\
K.W.Inch & 0.06 & 0.04 & 0.02 & 0.08 & 0.48 & 0.35 \\
\hline
\end{tabular}

ACKNOWLEDGEMENTS

This work has been partly funded by the EU project Hydralab+ (proposal number 64110), by theINTERREG V-A Italia-Malta 2014-2020 project NEWS - Nearshore hazard monitoring and Early Warning System (code C1-3-2-60), and by the ESF Operational Programme POR SICILIA FSE (CCI: 2014IT05SFOP014).

\section{References}

T. Aagaard, K. P. Black, and B. Greenwood. Cross-shore suspended sediment transport in the surf zone: a field-based parameterization. Marine Geology, 185(3):283-302, 2002.

F. Biesel. Second order theory of manometer wave measurement. In Coastal Engineering 1982, pages 129-135. 1982.

C. T. Bishop and M. A. Donelan. Measuring waves with pressure transducers. Coastal Engineering, 11(4): 309-328, 1987.

L. Cavaleri, J. Ewing, and N. Smith. Measurement of the pressure and velocity field below surface waves. In Turbulent fluxes through the sea surface, wave dynamics, and prediction, pages 257-272. Springer, 1978.

J. Chen. Three dimensional nonlinear pressure transfer function. Private Communication, 2000.

M. Hom-ma, K. Horikawa, and S. Komori. Response characteristics of underwater wave gauge. In Coastal Engineering 1966, pages 99-114. 1967.

K. Inch. Surf zone hydrodynamics: Measuring waves and currents. Geomorphological Techniques, 2014.

Y.-Y. Kuo and Y.-F. Chiu. Transfer function between wave height and wave pressure for progressive waves. Coastal Engineering, 23(1-2):81-93, 1994.

U. Neumeier. Processing of wave data from pressure sensors. http://neumeier.perso.ch/matlab/ waves.html, 2006.

U. Neumeier and C. L. Amos. The influence of vegetation on turbulence and flow velocities in european salt-marshes. Sedimentology, 53(2):259-277, 2006. 
K. L. Oliveras, V. Vasan, B. Deconinck, and D. Henderson. Recovering the water-wave profile from pressure measurements. SIAM Journal on Applied Mathematics, 72(3):897-918, 2012.

C. H. Tsai, M. C. Huang, F. J. Young, Y. C. Lin, and H. W. Li. On the recovery of surface wave by pressure transfer function. Ocean Engineering, 32(10):1247-1259, jul 2005. ISSN 00298018. doi: 10.1016/j.oceaneng.2004.10.020.

M. Tucker and E. Pitt. Waves in ocean engineering. 2001.

L. Van Rijn, B. Grasmeijer, and B. Ruessink. Measurement errors of instruments for velocity, wave height, sand concentration and bed levels in field conditions. ... /Delft Hydraulics Report, 0(November), 2000. 